\title{
DESARROLLO DEL TALENTO EN LA EDUCACIÓN FORMAL
}

\author{
Teresa Vinueza \\ Jonathan Esteban Castro Terán \\ Unidad Educativa Particular "Lev Vygotsky" (Ecuador)
}

\begin{abstract}
Resumen. Este artículo ofrece una revisión sobre los nuevos paradigmas de talento el cual no sólo se considera como un don de ciertos privilegiados, sino que puede ser desarrollado y potenciado con un entrenamiento adecuado. Presenta un innovador programa que sistematiza estrategias metodológicas para descubrir y desarrollar el talento dentro de la educación formal, y posteriormente vincularlo con una elección adecuada de la carrera universitaria por parte de los estudiantes. Se interrelacionan programas estudiantiles donde los educandos de Bachillerato (entre 16 y 18 años) lideran el proceso, reconociendo sus talentos para posteriormente desarrollarlos mediante un proyecto auto-propuesto en entornos de aprendizaje cooperativo con estudiantes de educación básica (entre 10 y 14 años). Para el análisis del impacto del programa según la percepción de los estudiantes, se utilizó una encuesta de 13 ítems, cuyos resultados fueron analizados con el paquete estadístico SPSS, obteniendo de esta manera un impacto medio de 4 sobre 5 puntos. Además, cabe recalcar que los estudiantes alcanzaron las herramientas necesarias e indispensables para una elección de la carrera universitaria basada en los talentos sobresalientes de cada uno.
\end{abstract}

Palabras clave: Talento, proyectos estudiantiles, elección de carrera universitaria.

\section{TALENT DEVELOPMENT IN FORMAL EDUCATION}

\begin{abstract}
This article offers a review about the new talent paradigms, according to which this is not considered as a gift of certain privileged people, but it could be developed and empowered with a proper training. This shows an innovator program that systematizes methodological strategies to discover and develop the talent within formal education, and then links it with a proper choice of a college degree by the students. In addition, there are interrelated student programs where high school students (between 16 and 18 years old) lead the process, acknowledging their talents to develop them later through a selfproposed project on learning environments with primary schoolers (between 10 and 14 years old). For the impact analysis according to the perception of the students, a 13 items poll was used, whose results were analyzed with the SPSS statistic program, getting this way an impact of 4 over 5 points. Furthermore, it's noteworthy that the students reached the necessary and indispensable tools to select a college degree based on each student's outstanding talent.
\end{abstract}

Keywords: Talent, student projects, selection of a college degree.

\section{DESENVOLVIMENTO DO TALENTO NA EDUCAÇÃO FORMAL}

Resumo. Este artigo oferece uma revisão dos novos paradigmas de talento, segundo os quais isso não é apenas considerado um dom de certos privilégiados, mas pode ser desenvolvido e maximizado com treinamento adequado. O resumo apresenta um programa inovador que sistematiza estratégias 
metodológicas para descobrir e desenvolver o talento dentro da educação formal e, posteriormente, vinculá-lo com uma escolha adequada da carreira universitária pelos alunos. Os programas estudantis estão inter-relacionados onde os alunos do ensino médio (entre 16 e 18 anos) lideram o processo, reconhecendo seus talentos e depois desenvolvendo-os através de um projeto autoproposto em ambientes cooperativos de aprendizagem com estudantes de educação básica (entre 10 e 14 anos). Para a análise do impacto do programa de acordo com a percepção dos alunos, foi utilizada uma pesquisa de 13 itens, cujos resultados foram analisados com o pacote estatístico SPSS, obtendo assim um impacto médio de 4 sobre 5 pontos. Além disso, é necessário enfatizar que os alunos alcançaram as ferramentas necessárias e indispensáveis para uma escolha da carreira universitária com base nos talentos de cada um.

Palabras-chave: talento, projetos estudantis, escolha de carreira universitária.

\section{Introducción}

La educación es un proceso dinámico en constante transformación que constituye uno de los fundamentos del desarrollo de una sociedad que prioriza el conocimiento y que requiere ciudadanos activos, creadores y emprendedores. Se puede responder a las exigencias de dicho desarrollo, solamente, teniendo como agentes activos a instituciones educativas responsables que, mediante la adopción de innovaciones, la implementación de nuevos modelos pedagógicos, la aplicación de metodologías actuales y permanente capacitación docente, procuren formar ciudadanos altamente competentes científica, técnica y humanísticamente. De ahí que, a mayor libertad educativa mayor desarrollo tendrá la sociedad, puesto que, de la competencia positiva que proporciona a niños y jóvenes una adecuada educación y una formación integral, se garantiza la base para combatir las injustas inequidades de origen social, económico o familiar.

El fin de la educación es liderar la formación integral de los niños y adolescentes, a través del perfeccionamiento y mejoramiento del desarrollo de los procesos educativos y desempeño docente; al igual que el cumplimiento de la función social de la institución educativa de acuerdo con las características, necesidades, intereses y expectativas de la comunidad circundante.

Las instituciones educativas tienen la responsabilidad de formar bachilleres con herramientas suficientes para sobresalir en el campo cognitivo y afectivo. Sin embargo, vemos con preocupación que la mayoría de estudiantes al llegar a su último año de Bachillerato no tienen definido su proyecto de vida laboral, pues no han elegido aún una carrera universitaria que esté acorde con sus fortalezas.

Por ello, se vuelve indispensable que dentro de la educación formal se profundice en procesos que permitan un eficaz desarrollo del talento con una estructura técnicamente diseñada, con propósitos claros y aprendizajes coherentes, que admitan que los estudiantes puedan direccionar sus aptitudes y actitudes destacadas hacia metas y objetivos personales, vinculando la carrera universitaria elegida con sus competencias sobresalientes convertidas en talentos.

La elección de una profesión permite abarcar, no solo una opción profesional, sino un perfil de vida, es por ello que esta decisión debe hacérsela con la conciencia de que permitirá formar la propia identidad, direccionará el rol que se debe asumir, establecerá un estatus y hasta se podrá concretar el proyecto de vida afectivo.

El talento no aparece como algo espontáneo, más bien sus primeros rasgos se presentan en la infancia e irán configurándose durante la pubertad y adolescencia, en 
donde, con una adecuada orientación y exploración de los mismos, podrán definirse, desarrollarse y tomar decisiones acertadas en la adultez.

En el Ecuador se han generado políticas públicas que establecen la importancia de intervenir en el desarrollo del talento; estas políticas plantean que desde las primeras etapas de escolarización se lo debe desplegar, ya que éste generará ciudadanos aptos para enfrentar los retos del nuevo milenio. La evolución de la ciencia y la tecnología afectan positivamente en la sostenibilidad de la economía presente y futura, fundamentando el progreso ecuatoriano, "bajo la única fuente inagotable de riqueza, el talento humano" (Correa, 2015, pág. 30)

Actualmente en la Malla Curricular ecuatoriana se presenta a "Proyectos Escolares" (eje interdisciplinario) y al "Programa de Participación Estudiantil" como gestores del descubrimiento y desarrollo de talentos mediante aprendizaje cooperativo; bajo esta premisa se estructura un proyecto estudiantil para el desarrollo del talento, que puede ser implementado en la educación formal y cuyos resultados se exponen en el presente documento.

\section{Nuevos paradigmas de talento}

La inteligencia desde el punto de vista de Binet es calificada como una variable de gran dependencia genética que será difícil modificarla a pesar de los esfuerzos que se puedan realizar mediante la educación (Mora \& Martín, 2007), considerando que el educando asume un rol pasivo cuyos comportamientos estables son impulsados por la interacción de factores intrapsíquicos (De Zubiría, 2004). Es por ello que, los test normalizados que tratan de medir la inteligencia desde esta óptica, únicamente juzgan una fracción de todas las habilidades que se necesitan para lograr el éxito dentro de la educación formal y sobre todo en la vida cotidiana.

Posteriormente aparecen nuevos planteamientos basados en Piaget (Medina, 2000) y Vygotsky (Rodríguez, 1999) que agregan factores ambientales a las bases teóricas del estudio de la inteligencia. El ambientalismo destaca la indiscutible trascendencia del conocimiento, así como el análisis de las situaciones contextuales que envuelven a las personas. Por otra parte, por el carácter activo de los seres humanos, el interaccionismo da la importancia al conocimiento de la situación de los escolares, pero en mayor grado a la interacción que se produce entre los elementos mencionados. De esta manera se empieza a reconocer los factores cognitivos y afectivos de los sujetos como elementos esenciales dentro del proceso de aprendizaje; además se observa a la persona como un integrante activo, que tiene la capacidad de modificarse y ser modificado a partir de los escenarios de aprendizaje con los que interactúa.

Es así que surge una variada terminología que procura tener un carácter más específico a fin de delimitar a la inteligencia superior; de este modo, dotación, talento y excepcionalidad, son términos empleados para definir a los sujetos que sobresalen en algún área (Tourón, 2004; Feldhusen, 2005).

Actualmente se impulsa establecer una diferencia entre los conceptos de dotación y talento, esto debido a que en un gran porcentaje de la literatura científica los han considerado como sinónimos, sin caracterizarlos para establecer sus diferencias y similitudes. A la dotación se la relaciona con una alta capacidad cognitiva, identificándola en mayor medida con aquellas personas que poseen un elevado coeficiente intelectual; por otra parte, el talento se distingue por considerarlo una aptitud sobresaliente que se manifiesta de manera espontánea en un área específica (deportes, artes, etc.) y que puede ser desarrollado. En ciertas ocasiones la dotación puede estar 
determinada como un nivel más alto en comparación con el talento, debido a que este requerirá de un proceso que permita alcanzar un alto nivel.

Las teorías acerca del génesis del talento son variadas, en un inicio se lo reconoce como una aptitud congénita de ciertos individuos, ocasionado por una dotación genética (Moreno, 1995), más tarde, y acorde a la evolución de los estudios sobre inteligencia (Coyle, 2009; De Zubiría, 2004; Gagné, 2015; Marina, 2016; Renzulli, 2008) se determina que los seres humanos nacen con un mínimo de aptitudes intelectuales, las cuales se pueden desarrollar a través de procesos de formación de habilidades, estimulación de las capacidades (como meta el aprendizaje), transferencia de los aprendizajes a las realidades cercanas y en mayor medida a través de conexiones neuronales que se producen dentro del cerebro (Coyle, 2009).

John Feldhusen, uno de los primeros investigadores que señala las diferencias entre talento y superdotación, manifiesta que el talento se percibe como aquellas "aptitudes especializadas crecientes o habilidades que desarrollan los jóvenes como una función de la habilidad general o inteligencia, y de sus experiencias educativas en casa, colegio y comunidad" (Feldhusen, 1995, p. 4), que se despliegan en función de las motivaciones e intereses que movilizan la voluntad de los individuos con el fin de especializarse en una actividad delimitada que requerirá una permanente práctica.

Abraham Tannenbaum (2015), luego de reconocer a los niños y adolescentes con un talento potencial, incluye en sus estudios los rasgos de personalidad, pero sobre todo las interacciones ambientales (desde el punto de vista socio-cultural) y elabora una teoría empírica a partir de cinco variables internas y externas, que combinadas provocan el desarrollo del talento.

A partir de esto, el talento se puede desarrollar en un ambiente propicio, ya que desde esta perspectiva aquellos que manifiestan capacidades superiores son considerados "potencialmente dotados" y el verdadero talento es "resultado de una compleja red de características innatas y medio ambiente" (Blumen, 2015, p. 226).

\section{Por su parte, Françoys Gagné (2007) señala que:}

El Modelo Diferenciado de Dotación y Talento (MDDT) se creó a partir de esa distinción, la que se constituyó en la base de nuevas definiciones distintivas de esos dos términos. DOTACIÓN designa la posesión y uso de capacidades naturales destacadas, llamadas aptitudes, en al menos un área o dominio de capacidad, en un grado que sitúa al individuo dentro del $10 \%$ superior de sus pares de edad. TALENTO designa el dominio destacado de capacidades sistemáticamente desarrolladas, llamadas competencias (conocimientos y destrezas), en al menos un campo de la actividad humana, en un grado que sitúa al individuo dentro del $10 \%$ superior de sus pares de edad que están o han estado activos en ese campo (Gagné, 2007, p. 2-3).

Para Gagné, tanto la dotación como el talento poseen cualidades comunes que las definen como las capacidades humanas que se ubican sobre el promedio general y que manifiestan conductas destacadas (Gagné, 2015). Sin embargo, la dotación está ligada con los "dones genéticos superiores", mientras que el talento es la transformación progresiva de dones (no siempre superiores) en talentos. En este último proceso los llamados "catalizadores intrapersonales y ambientales" afectarán de manera positiva o negativa al desarrollo del talento.

Por otra parte, Dan Coyle (2009) muestra una sencilla fórmula enfocada al desarrollo del talento de todas las personas que conjuga factores que permiten que el cuerpo produzca sustancias cerebrales y potencien la sinapsis neuronal. A partir de esto, 
tanto científicos como investigadores han centrado sus estudios en cómo se produce la mielina en el cerebro; dicha sustancia rodea el núcleo de las neuronas lo que faculta una comunicación neuronal ágil. Es así que el talento deja de ser una condición misteriosa, consecuencia del azar o la genética, y se la considera como cualidad que puede ser desarrollada partiendo de una alta motivación, a través de la práctica intensa y con la intervención de un maestro instructor.

Dicho instructor desarrolla el talento mediante:

- La ignición o motivación que brinda la energía indispensable para el desarrollo del talento;

- El impulso de la activación de los circuitos neuronales que corresponden al talento, y, por último;

- La práctica intensa que acciona el impulso nervioso y que mejora el circuito por medio de la rectificación de errores.

Así mismo, Robert Sternberg (1997) considera al talento como la inteligencia exitosa, calificándola como una habilidad intencionada que permite que los individuos se puedan adaptar a diversos ambientes, moldearlos y seleccionarlos; además tendrán la capacidad de alcanzar propósitos propios, para nuestra sociedad y para la cultura (Sternberg \& Galmarini, 1997). A esto se le conoce como "Teoría de la Inteligencia Exitosa" denominada también como Teoría Triárquica, ya que fusiona capacidades analíticas, creativas y prácticas, las mismas que al ser equilibradas maximizan los puntos fuertes para que se superen los débiles de cada individuo.

De la misma manera Joseph Renzulli (2017) declara que por medio de una intervención educativa se puede registrar los elementos relevantes que permiten que el ser humano alcance producciones creativas por medio del potencial que cada uno posee. En esta teoría se fusionan la capacidad intelectual, el compromiso en la tarea y la creatividad, vinculando estos elementos con las áreas de desempeño de cada persona.

Por otra parte, Tourón (2015) propone que "la capacidad humana es una suerte de combinación entre herencia y ambiente, y todos tenemos alguna capacidad, todos tenemos talento", es decir que los sujetos, con sus diferencias, poseen una capacidad que origina uno o varios talentos, los cuales deben ser desarrollados en función de sus facultades a fin de alcanzar su nivel máximo y el cumplimiento de sus metas.

Para José Antonio Marina (2010), la educación tiene el objetivo de desarrollar el talento de cada individuo y de la comunidad. Es por ello que la educación necesita una transformación por medio de la mediación, es decir, agenciar el aprendizaje permitiendo que los educandos se transformen en sus propios oficiales de desarrollo alcanzando la autonomía (Brito, 2017).

Debido a que muchos de los genes son activados a través de la educación y el entorno (Marina, 2010), la escuela se convierte en la mejor herramienta para gestionar el talento, para ello es necesario muchas horas de entrenamiento a fin de adquirir estructuras mentales que permitan el cumplimiento de la competencia y hábitos que la refuercen. Desde este punto de vista la inteligencia es considerada como la sinfonía de grandes fases que se complementan y de las cuales depende el éxito escolar, que va más allá de los resultados académicos y que más bien se enfoca en la transferencia de lo asimilado a la praxis enfocada en el beneficio propio y de los demás, es decir, "no solo se debe aprender a vivir, sino aprender a convivir" (Marina, 2010, p. 14). 
Los componentes de la inteligencia para Marina (2010, p. 14) son: Inteligencia Creadora, la misma que tiene la misión para satisfacer necesidades propias o ajenas; Inteligencia Ejecutiva, recibe las ideas resultado de la primera etapa, analizando la pertinencia y suprimiendo las que no sean útiles; y, por último, Inteligencia Evaluadora que determina cómo seleccionar las metas para lograr la felicidad personal, que está íntimamente ligada con la felicidad de los que nos rodean. De esta manera, el talento se entiende como la fusión entre la inteligencia creadora y la ejecutiva junto con la capacidad de autoevaluarse (Marina, 2010).

Dentro del Modelo de Pedagogía Conceptual existe un claro interés sobre desarrollo del talento planteándolo como uno de sus propósitos fundamentales. En este modelo se establece necesario realizar en los estudiantes exploraciones sistemáticas tanto de las motivaciones como de sus capacidades desde las edades tempranas, afirmando que la formación integral:

Exige explorar, identificar, fundamentar y canalizar el o los talentos de cada niño y niña, buscando hacerlo en un futuro el mejor trabajador posible: un TALENTO CREATIVO. Para bien de sí mismo y bien de los suyos, de su país, de su región. Tal propósito requiere explorar el talento de TODOS los pequeños desde los primeros cursos, identificarlos al iniciar la secundaria; fundamentarlo y canalizarlo pronto hacia un campo definido (De Zubiría, 2007, p. 11-12).

La propuesta radica en que todos los estudiantes al culminar con su bachillerato, ya habrán acumulado miles de horas de entrenamiento en el desarrollo de su talento.

Desde esta perspectiva podemos conceptualizar al talento por medio del siguiente mentefacto conceptual:

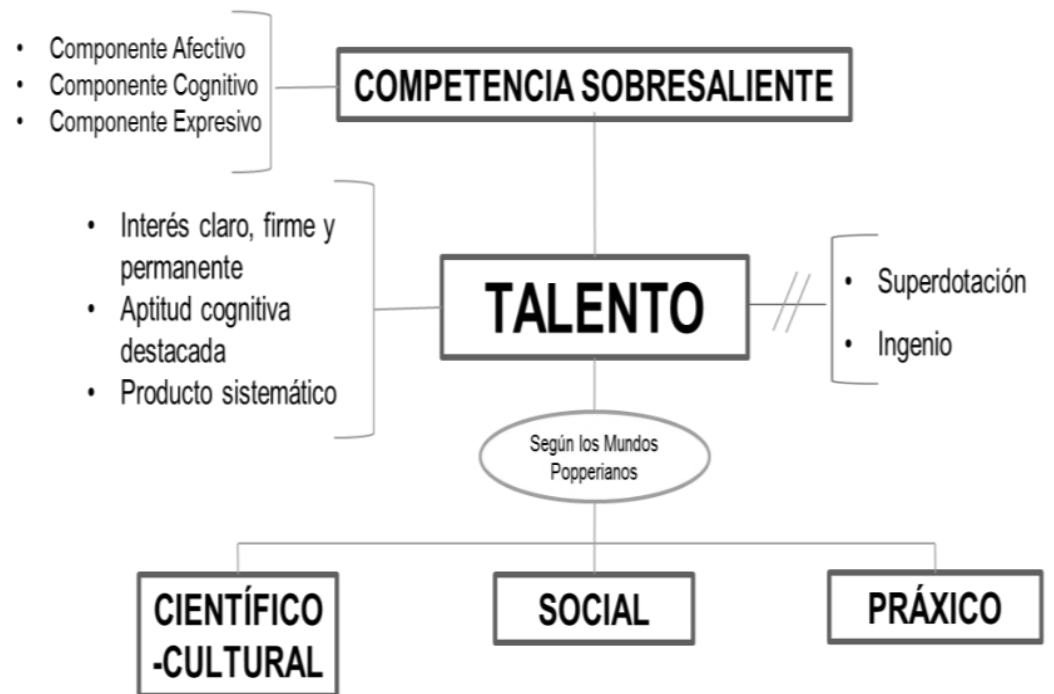

Figura 1. Mentefacto Conceptual de Talento. Elaborado por la Fundación Alberto Merani de Pedagogía Conceptual (2014)

Así como a la superdotación e ingenio, al talento se lo considera como una competencia sobresaliente que tienen los individuos en uno o varios campos. Esta condición estará definida en los componentes afectivo, cognitivo y praxitivo, diferenciándolo en la Tabla 1: 
Tabla 1

Comparación entre talento, superdotación e ingenio con respecto a los componentes del Triángulo Humano de la propuesta de Pedagogía Conceptual

\begin{tabular}{clll}
\hline \multirow{2}{*}{ TALENTO } & $\begin{array}{l}\text { Componente } \\
\text { Afectivo }\end{array}$ & $\begin{array}{l}\text { Componente } \\
\text { Cognitivo }\end{array}$ & $\begin{array}{l}\text { Componente } \\
\text { Praxitivo }\end{array}$ \\
\hline \multirow{2}{*}{ SUPERDOTACIÓN } & $\begin{array}{l}\text { Interés claro, } \\
\text { firme y } \\
\text { permanente }\end{array}$ & $\begin{array}{l}\text { Aptitud cognitiva } \\
\text { destacada en al } \\
\text { menos un área }\end{array}$ & $\begin{array}{l}\text { Productos } \\
\text { sistemáticos }\end{array}$ \\
& $\begin{array}{l}\text { Interés } \\
\text { espontáneo, } \\
\text { múltiple y } \\
\text { marcado }\end{array}$ & $\begin{array}{l}\text { Coeficiente } \\
\text { intelectual } \\
\text { superior }\end{array}$ & $\begin{array}{l}\text { Varias área de } \\
\text { predominio, } \\
\text { principalmente } \\
\text { lógico y verbal }\end{array}$ \\
\hline \multirow{2}{*}{ INGENIO } & $\begin{array}{l}\text { Interés focalizado } \\
\text { de alta intensidad } \\
\text { y corta duración }\end{array}$ & $\begin{array}{l}\text { Creatividad como } \\
\text { proceso } \\
\text { psicológico } \\
\text { superior }\end{array}$ & $\begin{array}{l}\text { Productos } \\
\text { prácticos y } \\
\text { vistosos }\end{array}$ \\
\hline
\end{tabular}

Nota: Elaboración propia

Por otra parte, las características exclusivas del talento se las define así:

- Interés claro, firme y permanente. - proceso que focaliza la motivación y atención de la persona en cosas o actividades específicas, permitiendo que se genere la motivación necesaria para dar inicio al aprendizaje.

- Aptitudes cognitivas destacadas. - se refiere a las disposiciones naturales que presentan las personas para una acción determinada, con ejercitación y entrenamiento se desarrollan y podrían convertirse en talento.

- Productos sistemáticos. - son soluciones (tangibles, virtuales, servicios, etc.) que se ponen a disposición para solventar necesidades; deben ser: sistemáticos, útiles, sobresalientes, reconocidos y replicables.

Finalizando, el talento, desde la perspectiva de Pedagogía Conceptual se clasifica de acuerdo a los Mundos Popperianos, es decir, concibiendo a la realidad como una trilogía de mundos (3M); el Mundo 1 (M1) de lo concreto; el Mundo 2 (M2) la realidad de los estados mentales y psicológicos; y el Mundo 3 (M3), realidad de los símbolos y creaciones humanas compartidas que configuran la ciencia y cultura (Popper, 1996). Es así que el talento se desarrollará dentro de uno de los mundos popperianos, siendo determinado por el nivel de competencia, incrementando su precisión al vincularlo con una o varias de las inteligencias que poseen los seres humanos (Gardner, 2001). Con su talento desarrollado los jóvenes obtendrán las herramientas necesarias para afrontar el mundo actual y futuro, globalizado, competitivo, con un sin número de instrumentos tecnológicos y avances científicos constantes, que ofrecerá grandes oportunidades a quienes tengan la capacidad de utilizarlos.

\section{Descripción del programa estudiantil para el desarrollo del talento}

El programa estudiantil se llevó a cabo durante dos años lectivos en la Unidad Educativa Particular "Lev Vygotsky" ubicada en Sangolquí-Ecuador. En el primer año 
se capacitó a los estudiantes que iniciaban su bachillerato sobre talento (conceptualización y exploración), aprendizaje cooperativo y desarrollo de proyectos; en el segundo año, los estudiantes capacitados, asumieron la dirección de un grupo de estudiantes de educación básica (diferentes niveles) para desarrollar un proyecto autopropuesto relacionado con su talento e inteligencia predominante. El objetivo del programa es demostrar la importancia que tiene el desarrollo del talento en los estudiantes, y como éste puede ser estimulado mediante el trabajo cooperativo entre compañeros de bachillerato y educación básica, además el talento desarrollado servirá de guía para elegir una carrera universitaria afín a las potencialidades propias (Tabla 2).

Tabla 2

Estructura del Programa de Participación comparando los contenidos abordados con las fases con la metodología utilizada.

\section{DESCRIPCIÓN}

Propósito: Fomentar un ambiente favorable para alcanzar los objetivos de aprendizaje planteados.

Necesidad Pedagógica: Autorregulación estudiantil.

Enseñanzas:

-Reglas y normas a seguir.

-Bases legales del Programa de Participación generadas por el

INICIO Ministerio de Educación.

Persigue la

disonancia del sistema cognitivo y

lleva al sistema afectivo a comprometerse con el aprendizaje.

Meta: Estudiantes conscientes de las normas Institucionales y Ministeriales

Propósito: Comprender la necesidad de aprender sobre el talento para que exista una vinculación afectiva con este nuevo aprendizaje.

Necesidad Pedagógica: Valoración de la importancia del desarrollo del talento.

Enseñanzas: Argumentos de la importancia de trabajar sobre los talentos personales.

Meta: Estudiantes reflexivos frente a la importancia de los aprendizajes que se impartirán en el Programa.

DESARROLLO Propósito:

-Conceptualizar el talento.

Lleva a cabo las acciones de instrucción e interacción que le permitan al sistema cognitivo reacomodarse y

-Distinguir nociones elementales del aprendizaje cooperativo. -Conocer los pasos básicos para la elaboración de proyectos sencillos.

Necesidad Pedagógica:

-Razona sobre los elementos del concepto de talento.

-Establece lineamientos básicos para el trabajo cooperativo.

-Reconoce los elementos que debe tener su proyecto. 
reorganizarse.

Para esto, el educador utiliza información estructurada en diferentes instrumentos de conocimiento.
Enseñanzas:

Instrumentos del conocimiento. -

- Concepto de Talento

-Argumentos sobre aprendizaje colaborativo.

-Procedimiento para elaborar un proyecto.

Meta: Estudiantes que comprenden los instrumentos del conocimiento abordados y sean capaces de ejemplificarlos.

Propósito: Asimilar las enseñanzas impartidas a partir de la observación de casos.

Necesidad Pedagógica: Comprende los conceptos, argumentos y procedimientos que se establecen en el Programa.

Enseñanzas:

Aprendizajes aplicados en situaciones recreadas.

- Concepto de talento: Analizar la vida de personas talentosas.

- Argumentos de aprendizaje cooperativo: Observar situaciones reales.

-Elaboración de un proyecto: Analizar proyectos ya elaborados.

Meta: Estudiantes que comprenden las características y procedimientos de las enseñanzas impartidas.

Propósito: Concientizar aciertos y errores desde una práctica guiada.

Necesidad Pedagógica: Admite el nivel de apropiación de los aprendizajes y supera las dificultadas.

Enseñanzas:

Análisis de recomendaciones.

- Concepto de talento: Exploración de talentos.

- Argumentos de aprendizaje cooperativo: Realizar actividades colaborativas y concientizar errores en la ejecución de roles.

-Elaboración de un proyecto: Elaboración de un primer borrador.

Meta: Estudiantes que monitoreen y concienticen la aplicación práctica de los nuevos aprendizajes.

Propósito: Desarrollar autónomamente las actividades planteadas.

Necesidad Pedagógica: Realiza actividades sin necesidad de la guía del profesor.

Enseñanzas:

Aprendizajes aplicados en situaciones reales.

- Concepto de talento: Aplicación del talento en la elaboración de proyectos.

- Argumentos de aprendizaje cooperativo: Liderazgo de grupos de trabajo con estudiantes de diferentes niveles. 
-Elaboración de un proyecto: Elaboración y ejecución del proyecto planteado.

Meta: Estudiantes que transfieran sus aprendizajes a situaciones reales.

Propósito: Evaluar la calidad de los aprendizajes alcanzados.

Necesidad Pedagógica: Concientiza sus niveles de desempeño.

\section{CIERRE}

Permite que a través de diferentes acciones se lleve al estudiante a manejar con autonomía las nuevas redes cognitivas que posee para una transferencia a la realidad.

\section{Enseñanzas:}

-Reconocimiento de su talento.

-Autoevaluación de su liderazgo en la guía del grupo de trabajo. -Análisis del producto final de su proyecto.

Meta: Estudiantes conscientes de su nivel de apropiación de las enseñanzas que generó el programa.

Propósito: Sintetizar las experiencias cognitivas y afectivas que determinaron el éxito o fracaso del programa.

Necesidad Pedagógica: Reconoce su talento y la importancia de desarrollarlo a través de una metacognición.

Enseñanzas:

Resumen de todos los aprendizajes alcanzados.

Meta: Estudiantes que utilicen los nuevos aprendizajes en situaciones cotidianas de su vida fuera del colegio.

Nota: Elaboración propia

\section{Metodología}

\section{Diseño de la Investigación}

La presente investigación se ha realizado bajo un enfoque metodológico de tipo cuantitativo, esto se debe a que el principal objetivo de este estudio es demostrar que, con un programa de exploración de talentos desarrollado como parte de la educación formal, los estudiantes adquieren mayores y mejores elementos para una adecuada elección de su carrera universitaria. Es necesario comprender, bajo la experiencia vivida, qué factores intervienen para la adecuada selección de una carrera de tercer nivel tomando como base el reconocimiento y desarrollo del talento por parte de los estudiantes.

\section{Participantes}

En el estudio participaron 49 estudiantes de Segundo Año de Bachillerato, divididos en dos cursos, quienes iniciaron el programa desde el año 2016, distribuidos en dos paralelos ( 27 hombres y 22 mujeres). La edad media de los participantes fue de 16,84 años $(S D=0,36)$, pues el rango de edades varió entre 16 años y 18 años.

La muestra estuvo conformada por 25 estudiantes (11 mujeres y 14 hombres), los mismos que pertenecían a uno de los cursos seleccionado de manera aleatoria, 
representando así el 50\% de los estudiantes del nivel. Los estudiantes fueron notificados sobre su participación en el proyecto, luego de haber obtenido las respectivas aprobaciones de las autoridades de la institución.

\section{Instrumentos de recolección de información}

Se empleó una encuesta cuyo cuestionario constó de 13 preguntas, de las cuáles las 10 primeras eran preguntas cerradas y medidas en escala de valoración de 5 puntos. En la sección inicial se analizó la percepción de los estudiantes sobre el impacto inmediato que tuvo el Programa de Participación Estudiantil "Desarrollando el talento mediante el trabajo cooperativo autorregulado", tomando al 1 como el puntaje más bajo y 5 el puntaje el más alto; las cuestiones a averiguar se aprecian en la Tabla 3.

Tabla 3

Ítems para la evaluación de la percepción del impacto inmediato del programa

\section{Cuestión}

Item1 Un impacto positivo dentro de tu proceso de formación, al permitirte aprender sobre temas que no se encuentran dentro del currículo.

Item2 Oportuno en los conceptos y definiciones que se desarrollaron en él.

Item3 Adecuado en el planteamiento de ejercicios para que se logren alcanzar los objetivos planteados.

Preciso en la estructuración de actividades para la exploración de

Item4 talentos y elaboración de tu proyecto escolar.

Item5 Consistente al desarrollar en ti competencias afectivas sólidas que puedan ser replicadas en tu vida.

Pertinente como insumo para la elaboración de tu proyecto de vida

Item6 laboral.

Sólido en su ejecución y acompañamiento durante el proceso de

Generador de vínculos sociales cálidos dentro de la ejecución de las

Item8 actividades grupales.

Item9 Sistemático en el proceso de exploración de tu talento, con actividades adecuadas y oportunas.

Item10 Concluyente para tu autoconocimiento y definición de tu talento.

Nota: Elaboración propia

La segunda parte del cuestionario consistía en tres preguntas cerradas (Tabla 4), que permitieron cotejar la apreciación de los estudiantes sobre la influencia del talento explorado y desarrollado en la selección de la carrera universitaria; estos datos tuvieron un tratamiento diferente a los recolectados en la primera parte del instrumento. La cuestión uno era un reactivo de doble alternativa: SI - NO; sin embargo, para las dos últimas preguntas se empleó una escala estimativa con tres niveles: Bajo - Moderado Alto. 
Tabla 4

Ítems de la influencia del programa en la elección de la carrera universitaria

\section{Cuestión}

Item1 En la fase inicial del Programa al responder la primera encuesta, ¿tenías identificado un talento o inteligencia predominante?

Item2 Durante el proceso de exploración de tus talentos, ¿en qué medida desarrollaste un talento a través del Programa de Participación Estudiantil?

¿La carrera universitaria que has elegido para estudiar al culminar tu

Item3 bachillerato tiene relación con el talento desarrollado?

Nota: Elaboración propia

\section{Análisis de Datos}

Para analizar la confiabilidad del instrumento se consideró las medidas de consistencia interna las cuales requieren únicamente de una administración del instrumento. Esta aplicación fue hecha a un grupo de doce estudiantes de similares características a las del grupo de estudio.

Con los resultados tabulados se utilizó el coeficiente del Alfa de Cronbach, apoyado en el programa estadístico SPSS (versión 23.0.0.2), dando como resultado $\alpha=$ 0.834. Esto demuestra un nivel de confiabilidad aceptable, y por lo tanto certificó que los datos recolectados fueron consistentes y coherentes. Además, se realizaron análisis descriptivos de los datos para proceder a interpretarlos y posteriormente verificar si el proyecto alcanzó el impacto esperado.

\section{Procedimiento}

Para asegurar la efectividad del proyecto, éste se desarrolla en tres fases, una teórica enfocada en la conceptualización, la segunda dirigida a la ejecución de un proyecto auto-propuesto y la tercera direccionada a la evaluación del proyecto.

\section{Fase de Conceptualización.}

Para iniciar esta etapa los estudiantes llevan a cabo un test sobre las ocho inteligencias basado en la Teoría de Inteligencias Múltiples (Gardner, 2001) que fue elaborado y validado para el proyecto, a fin de que puedan identificar su inteligencia y/o el talento predominante.

Esencialmente en la conceptualización se accionan dos sesiones semanales de 40 minutos cada una, durante un año escolar (diez meses); en estas clases se utiliza una guía didáctica para el estudiante, donde se encuentra la conceptualización de talento, junto con ejercicios prácticos que permiten una exploración sistemática del mismo en función de la conceptualización analizada; en las actividades planteadas se vinculan a las carreras universitarias que tienen relación con las clases de inteligencia o talento.

\section{Fase de Ejecución}

Durante este período, luego de haber identificado y desarrollado el talento, los jóvenes se plantean un proyecto para beneficio de la comunidad educativa que genere un producto; el mismo que debe ser elaborado y puesto en marcha a lo largo del siguiente año escolar durante sesiones de 60 minutos semanales. En este ciclo, cada estudiante de segundo de bachillerato lidera un grupo heterogéneo entre 8 y 10 
miembros de diferentes niveles escolares, a fin de alcanzar el objetivo que se habían planteado. Para el cierre de esta etapa los estudiantes realizan una exposición de su proyecto mostrando el producto final a la comunidad educativa.

\section{Fase de Evaluación}

En esta fase se analizan los ejercicios que cada uno de los estudiantes realiza en su guía didáctica, con el objetivo de examinar el proceso para identificar y desarrollar el talento; pero, sobre todo, este estudio se enfocó en comprobar si este talento que han definido como predominante tiene una estrecha correspondencia, o no, con los resultados del test inicial.

Además, se emplea un segundo test para averiguar la apreciación de los estudiantes sobre el impacto inmediato del Programa, permitiendo de esta manera corroborar que el talento y/o inteligencia sobresaliente está vinculado con la elección de la carrera universitaria.

\section{Resultados}

Se analizaron los estadísticos descriptivos (Tabla 5) para determinar la valoración ejecutada por los colegiales respecto a la percepción del impacto inmediato del programa, obteniendo un nivel alto $($ media $=4,00)$ y cercana al punto máximo de la tabla (5 puntos).

Tabla 5

Descriptivos satisfacción del programa

\begin{tabular}{cccccc}
\hline & N & Mínimo & Máximo & Media & $\begin{array}{c}\text { Desviació } \\
\text { n estándar }\end{array}$ \\
\hline Item1 & 25 & 1,00 & 5,00 & 3,9600 & 0,97809 \\
Item2 & 25 & 2,00 & 5,00 & 4,0800 & 0,81240 \\
Item3 & 25 & 2,00 & 5,00 & 3,6000 & 0,86603 \\
Item4 & 25 & 3,00 & 5,00 & 4,1600 & 0,74610 \\
Item5 & 25 & 3,00 & 5,00 & 4,2000 & 0,76376 \\
Item6 & 25 & 3,00 & 5,00 & 4,0800 & 0,75939 \\
Item7 & 25 & 2,00 & 5,00 & 3,6800 & 0,74833 \\
Item8 & 25 & 3,00 & 5,00 & 4,1600 & 0,68799 \\
Item9 & 25 & 3,00 & 5,00 & 4,1200 & 0,66583 \\
Item10 & 25 & 3,00 & 5,00 & 3,9600 & 0,73485 \\
Media Total & & & & 4,0000 & \\
\hline
\end{tabular}

Nota: Elaboración propia

Por otra parte, luego del análisis del segundo segmento del instrumento, los resultados muestran que el Programa de Participación Estudiantil "Desarrollando el talento mediante el trabajo cooperativo autorregulado" se relaciona positiva y significativamente con el desarrollo del talento, desde la perspectiva de los estudiantes, como se muestra en la Tabla 6. 
Tabla 6

Correlaciones entre desarrollo del talento y elección de la carrera universitaria

\begin{tabular}{ccccc}
\hline & & & Item12 & Item13 \\
\hline & Item1 & Coeficiente de & 1,000 & $0,467^{*}$ \\
& 2 & correlación & & 0,19 \\
Rho de Spearman & & Sig. (bilateral) & $\cdot$ & 0 \\
& \multirow{3}{*}{ Item1 } & N & 25 & 25 \\
& 3 & Coeficiente de & $0,467 *$ & 1,000 \\
& correlación & 0,19 & $\cdot$ \\
& Sig. (bilateral) & N & 25 & 25 \\
\hline
\end{tabular}

Nota: La correlación es significativa en el nivel 0,05 (bilateral). Fuente: Elaboración propia

\section{Conclusiones}

El presente estudio determinó el grado de satisfacción del programa de participación estudiantil que se efectuó con 25 estudiantes de segundo de bachillerato en una institución educativa ecuatoriana durante dos años lectivos, el cual gestiona al talento como insumo idóneo para la elección de la carrera universitaria. Según estudios de José Antonio Marina (2014), se afirma que no solo el período entre los 0 a 4 años de edad es el privilegiado para aprender o desarrollar un talento, sino que existe una segunda oportunidad en la adolescencia que se produce entre los 15 y los 18 años; en esta nueva coyuntura el cerebro del adolescente se rediseña.

Las fortalezas que se determinaron a lo largo del programa fueron:

- La educación formal es la responsable de gestionar espacios para el desarrollo del talento y estimular la capacidad del adolescente para plantearse metas coherentes, gestionando sus emociones para alcanzarlas.

- Los estudiantes pudieron conceptualizar al talento y vincular las características de éste con sus competencias sobresalientes, intereses, aptitudes y áreas de conocimiento.

- Las actividades propuestas permitieron que los estudiantes logren vincular sus inteligencias sobresalientes con un talento determinado para proceder a desarrollarlo mediante un proyecto auto propuesto.

- Se tomó al talento desarrollado como insumo para la elección de la carrera universitaria, facilitando a los estudiantes relacionarla con sus competencias sobresalientes. En el estudio se pudo determinar que, en el grupo examinado, existe una relación positiva y significativa entre el talento desarrollado y la elección de la carrera universitaria. siguientes:

Las limitaciones que se encontraron en la ejecución del programa fueron las

- Los talleres de capacitación sobre talento se desarrollaban solo los días viernes en las últimas horas de clase, situación que produjo que en reiteradas ocasiones se deban suspender por eventos institucionales o vacaciones gubernamentales. 
- Algunos estudiantes no dieron la relevancia necesaria a las actividades propuestas, al no tener una carga académica ni calificaciones numéricas, situación que provocó que el programa no tenga un impacto significativo en sus vidas.

Con estos elementos, podemos plantear las siguientes propuestas de mejora:

- $\quad$ Es necesario que se incorpore en los programas educativos y/o en los currículos institucionales al desarrollo del talento como un eje primordial para la orientación vocacional de los estudiantes, con la finalidad de que puedan estructurar un proyecto de vida coherente a su realidad y evitar la deserción universitaria causada por una inconsistencia entre el talento y la carrera elegida.

- El programa debe ser replicado en otras instituciones educativas con un mayor número de estudiantes y medir nuevamente su nivel su impacto.

- $\quad$ Es importante que en futuras aplicaciones del proyecto se vincule a los padres con actividades compartidas para que se conviertan en guía permanente de sus hijos en el descubrimiento y desarrollo del talento.

- $\quad$ Es necesario que se realice un seguimiento a una muestra representativa de los participantes durante sus estudios universitarios, midiendo nuevamente la correspondencia de la carrera universitaria y el talento o inteligencia predominante determinados en el programa.

\section{Referencias}

Amstrong, T. (2006). Inteligencias múltiples en el aula. Barcelona: Paidós.

Blumen, S. (2015). Abraham Tannenbaum: 1924 - 2014. Revista de Psicología, 33(1), 231-234.

Bravo, D. (2009). Desarrollo de la creatividad en la escuela. (1 ed.). San José: Colección Pedagógica Formación Inicial de Docentes.

Brito, J. (16 de Mayo de 2017). Pedagogía del desarrollo. (T. Vinueza, Entrevistador)

Bruner, J. (1988). Realidad mental y mundos posibles. Barcelona: Gedisa.

Cardozo-Ortiz, C. (2011). Tutoría entre padres como estrategia universitaria. En C. Cardozo-Ortiz, Tutoría entre padres como estrategia universitaria (págs. 3093025). Bogotá: Facultad de Educación - Universidad de la Sabana.

Castillo, A. (1997). Apuntes sobre Vygotsky y el aprendizaje cooperativo. Lev Vygotsky y sus aportaciones para el siglo XXI, 1, 47-57.

Comunidad de Madrid. (2012). Obtenido de Laboratoio de Innovación educativa: http://www.madrid.org/dat_capital/upe/impresos_pdf/AprendizajeCooperativo20 12.pdf

Correa, R. (2015). Encuentro Latinoamericano Progresista - II ELAP. Conferencia Magistal (págs. 7-37). Quito: Casa de la Cultura Ecuatoriana.

Coyle, D. (2009). Las claves del talento. Bogotá: Zenith.

De Zubiría. (2004). El mito de la inteligencia. Bogotá: Fundación Internacional de Pedagogía Conceptual. 
De Zubiría, M. (2006). Psicología del Talento y la Creatividad. Bogotá: Fundación Internacional de Pedagogía Conceptual.

De Zubiría, M. (2007). Introducción a la Pedagogía Conceptual. Congreso Latinoamericano de Estudiantes de Psicología (págs. 1-13). Bogotá: COLAEPSI.

Feldhusen, J. (1995). Identificación y desarrollo del talento en la escuela (TIDE). Ideacción, 4.

Feldhusen, J. (2005). Conceptions of Giftedness. En R. Sternberg, \& J. Davidson, Giftedness, Talent, Expertise, and Creative Achievement (págs. 64-80). New York: Cambridge University Press. doi: 10.1017/CBO9780511610455.006

Gagné, F. (2007). Construyendo talentos a partir de la dotación: Breve revisión del MDDT 2.0. Obtenido de http://www.eurotalent.org/MDDT_2.0_SP_overview.pdf

Gagné, F. (2015). From genes to talent: the DMGT/CMTD perspective. Revista de Educación, 368(1), 12-39. doi:10.4438/1988-592X-RE-2015-368-289

Gardner, H. (2001). Teoría de las inteligencias múltiples. Bogotá: Fondo de Cultura Económica.

Gutierresz-Braojos, C., Salmeron, H., \& Muñoz de Escalona, M. (2009). El aprendizaje autorregulado y las concepciones de los estudiantes en el aprendizaje cooperativo. Revista de Educación de la Universidad de Granada, 22(2), 73-82.

Johnson, D., Johnson, R., \& Holubec, E. (1999). El aprendizaje cooperativo en el aula. Barcelona: Paidos.

Marina, J. (2010). La educación del talento. Barcelona: Ariel.

Marina, J. (2012). La Inteligencia Ejecutiva. Barcelona: Ariel.

Marina, J. (2014). El talento de los adolescentes. Barcelona: España.

Marina, J. (2016). Objetivo: Generar talento. Barcelona: Conecta.

Medina, A. (2000). El legado de Piaget. Educere, 3(9), 10-15.

Mora, J., \& Martín, M. (2007). La Escala de Inteligencia de Binet y Simon (1905) su recepción por la Psicología posterior. Revista de la Historia de la Psicología, 28(2), 307-313.

Morejón, J. (2011). Fomentado los talentos en el aula. Sinaloa: Subsecretaría de Educación Básica.

Moreno, M. (1995). La determinación genética del comportamiento humano. Una revisión crítica desde la filosofía y la genética de la conducta. Gazeta de Antropología, 11.

Popper, K. (1996). En busca de un mundo mejor. Barcelona: Paidós.

Pujolas, P. (2008). 9 Ideas claves. El aprendizaje cooperativo. Barcelona: Grao.

Ramdass, D., \& Zimmerman, B. J. (2011). Developing Self-Regulation Skills: The Important Role of Homework. Journal of Advanced Academics, 22(2), 194-228. doi: $10.1177 / 1932202 X 1102200202$ 
Renzulli, J. (2008). La educación del sobredotado y el desarrollo del talento para todos. Revista de Psicología, 26(1), 26-41.

Renzulli, J. (17 de enero de 2017). Renzulli Center for Creativity, Gifted Education, and Talent Development. Obtenido de Renzulli Center for Creativity, Gifted Education, and Talent Development: http://gifted.uconn.edu/

Rodríguez, W. (1999). El legado de Vygotski y de Piaget a la educación. Revista Latinoamericano de Psicología, 31(3), 477-489.

Salmeron, H., \& Gutierrez-Braojos, C. (2012). La competencia de aprender a aprender y el aprendizaje autorregulado. Profesorado. Revista de Currículum y Formación de Profesorado, 16(1), 5-13.

Salmerón, H., Gutierrez-Braojos, C., Fernández, A., \& Salmeron, P. (2010). Aprendizaje autorregulado, creencias de autoeficacia y desempeño en la segunda infancia. RELIEVE. Revista Electrónica de Investigación y Evaluación Educativa, 16(2), 1-18.

Salmeron, H., Gutierrez-Braojos, C., Rodríguez, S., \& Salmerón, P. (2010). Influencia del aprendizaje cooperativo en el desarrollo de la competencia para aprender a aprender en la infancia. REOP - Revista Española de Orientación y Psicopedagogía, 21(2), 308-319. doi: 10.5944/reop.vol.21.num.2.2010.11534

Salmeron, H., Rodriguez, S., \& Gutierrez-Braojos, C. (2010). Methodologies to Improve Communication in Virtual Learning Environments. [Metodologías que optimizan la comunicación en entornos de aprendizaje virtual]. Comunicar, 34, 163-171. doi: 10.3916/C34-2010-03-16

Salmeron, H., Rodriguez, S., \& Gutierrez-Braojos, C. (2011). Metas de logro, estrategias de regulación y rendimiento académico en diferentes estudios universitarios. Revista de Investigación Educativa, 29(2), 467-486.

Salmeron, H., Rodriguez, S., Gutierrez-Barojos, C., \& Salmeron , P. (2010). Influencia del aprendizaje cooperativo en el desarrollo de la competencia para aprender a aprender en la infancia. Revista Española de Orientación y Psicopedagogía, 21(2), 308-319. doi: 10.5944/reop.vol.21.num.2.2010.11534

Sánchez-Bayo, A. (2010). Arquelogía del talento. Madrid: ESIC.

Sternberg, R. (2003). Wisdom, Intelligence, and Creativity Synthesized. London: Cambridge University Press. doi: 10.1017/CBO9780511509612

Sternberg, R., \& Galmarini, M. (1997). Inteligencia existosa. Barcelona: Paidós.

Tourón, J. (2004). De la superdotación al talento: Evolución de un paradigma. En Pedagogía Diferencial. Diversidad y Equidad (págs. 369-400). Madrid: Pearson Educación.

Tourón, J. (16 de marzo de 2012). Talento, ¿de qué hablamos? Obtenido de http://www.javiertouron.es/2012/03/talento-de-que-hablamos.html

Tourón, J. (27 de julio de 2015). Más mitos sobre la alta capacidad, aunque parezca imposible. Obtenido de http://www.javiertouron.es/2015/07/mas-mitos-sobre-laalta-capacidad.html

Tourón, J., \& Santiago, R. (2015). El modelo Flilpped Learning y el desarrollo del talento en la escuela. Revista de Educación(368), 174-195. 
Fecha de recepción: 03/10/2017

Fecha de revisión: 11/04/2018

Fecha de aceptación: 15/05/2018 